\title{
Research on Intelligent Library Document Navigation System Based on GIS
}

\author{
RuixiangOu ${ }^{1}$, Hui Pan ${ }^{1}$, Yao Huang ${ }^{2}$ \\ ${ }^{1}$ South China University of Technology Library, Guangzhou, 510640, China \\ ${ }^{2}$ Propaganda Department of Party Committee, Southern Medical University, Guangzhou 510515, \\ China
}

Key words: GIS ; library; intelligent literature navigation

\begin{abstract}
The intelligent navigation mode based on GIS technology uses the visualization positioning technology to locate the specific collection information of the literature to each layer of the bookshelf, and to display the information of the library by the way of the GIS . The readers do not need to master the literature order of the library; you can quickly access the required literature. The intelligent navigation mode fully exploits the potential of RFID technology, ArcGIS technology and Sharp Map technology, and constructs the library intelligent document navigation system.The application of the system in the library can further improve the library document management and information service efficiency, strengthen the library information service function, and strengthen the library information security center status.
\end{abstract}

\section{Introduction}

In recent years, with the increase of the total amount of library resources and the increase of readers, the traditional library literature navigation mode has been unable to provide readers with fast and efficient information navigation service, it need more intelligent document navigation system to meet the reader's information navigation needs.It is found that the navigation mode based on GIS technology has the characteristics of visibility, interactivity, fast and efficient, and it is very suitable for the design of intelligent library literature navigation system. This represents the future development direction of intelligent library literature navigation system.

\section{Overview of related technologies of intelligent library literature navigation system}

RFID technology is the abbreviation of Radio Frequency Identification, which use RF signal through the spatial coupling to achieve non-contact information transmission.In the intelligent library literature navigation system, RFID technology is mainly used for the tag of GIS data.Open source GIS database ArcGIS is an extension of PostgreSQL, an object-relational database system that provides spatial information services such as spatial objects, spatial indexes, spatial operations functions, and spatial operators, and conforms to OpenGIS specifications.In the intelligent library literature navigation system, ArcGIS is mainly used for storing, calculating and extracting GIS data.OpenMap library SharpMap is based on the. NET2.0 platform using C \# developed Map rendering class library, you can render ESRIShape and ArcGIS format GIS data, support B / S and C / S development application model.In the intelligent library literature navigation system, SharpMap provides technical support for visualizing the display GIS data, and enhances the system's visibility, mobility and interactivity. 


\section{Design of Document Navigation Mode for Intelligent Library Based on Open Source GIS Technology}

(1) The traditional library literature navigation mode

There are several procedures for the traditional library literature navigation mode:1.Give Literature library of each library a name.2.Make a book layout.3.Process the literature and assign it to the designated library.4.Readers use the library literature navigation system to query the literature collection information.5.Readers through the literature collection information to find documents resources.

In the traditional navigation mode, the reader finds the corresponding library, and then finds the literature according to the library collation rules.The survey shows that new readers can clearly understand the library layout according to the library layout.But the reader does not understand the rules of the library literature, the reader quickly find the literature is very difficult, affecting the reader's literature access efficiency.

(2) The intelligent library navigation mode

Intelligent library based on open source GIS technology Intelligent navigation mode includes several programs:1.Assign each function area of the library to a unique number.2.Make library main entrance as the reference coordinate point, markthe library function area, and then give its unique number.3.Paste the RFID tag on the outside of each document shelf in the library.4.Process the literature, and distribute the literature to the library.5.Develop Intelligent Library Document Navigation System.6.Readers access the literature through the Intelligent Library Document Navigation System.

The intelligent navigation mode based on GIS technology uses the visualization positioning technology to locate the specific collection information of the literature to each layer of the bookshelf, and to display the information of the library by the way of the GIS . The readers do not need to master the literature order of the library; you can quickly access the required literature.

\section{Research on Intelligent Library Document Navigation System Based on Open Source GIS Technology}

The Intelligent Library Document Navigation System is the software running platform which selects C \# language, uses the open source GIS database ArcGIS as the database management system, and the windows series operating system.

(1)The overall structure of intelligent library literature navigation system

Intelligent library document navigation system make maximize the advantages of GIS in the literature navigation, combined with RFID technology design based on open source GIS library SharpMap and open source GIS database ArcGIS intelligent document navigation system.

(2)Thebasic data processing module

The basic data processing module is divided into the function area number setting according to the system function positioning requirement, the function area is composed of the coordinate data acquisition, the document library frame coordinate data acquisition, the literature and the frame correspondence data acquisition 4 smaller functions Modules, these modules work together efficiently to complete the basic data processing tasks.

The setting number module will be the library of the functional areas by the order of the allocation of different numbers, the Chinese region name and number corresponding to the data stored in the database. The relevant database table (each function area number data table) includes three fields, namely, the function area number, the function area Chinese name, the area where the 
function area is located.

The coordinate data acquisition module marks the coordinate information of the key components of each functional area with reference to the library design and drawing, and stores the data into the database. The relevant database table (function area key composition point coordinate data table) includes 5 fields, namely point number, point abscissa, point ordinate, function area number, function area where the floor.

The Library frame coordinate data acquisition moduletakes the information such as the coordinates, layer height and layer number of the four layers of the bookshelves of the library, and puts the data into the database. The relevant database table (the composition of the library coordinates of the data table) includes five fields, namely, layer number, layer height, point number, point abscissa, point vertical axis.

The Document and Frame Correspondence Data Acquisition Module will be associated with the frame, the data into the database. Related database tables includetwo fields, namely, document number, layer number.

\section{The intelligent document navigation module}

Intelligent document navigation module according to the system function positioning needs, subdivided into a variety of plane layout diagram display module, the precise positioning of the literature frame and schematic display module and literature frame document management module 3 smaller functional modules, the three functional modules efficient operation, together to complete the intelligent document navigation task.

A variety of flat layout schematic display module shows the library floor of the library function area location plane layout diagram. The reader can open the intelligent document navigation system software click on a floor to view the floor function area location plan, can also use the functional area location search function to find the specified function area location diagram.

The precise positioning of the frame and the presentation module are the core of the system. Readers use the library's existing retrieval system to find the required literature collection information, if the literature can copy the literature number, and then open the system, in the literature search function area enter the literature number and click on the search, the system in the form of plane position will The precise location of the literature (including the high level of information) to show in front of the reader, the reader can quickly according to the location of the literature in place to access the literature. The system shows the reader's schematic to zoom in, zoom out and move, giving the reader a new app experience.

The document frame management function can be used to assist the administrator in organizing the literature library literature. The administrator uses the RFID tag reader connected to the system to read the document shelf document storage unit tag and the document storage unit document label, the system can display the literature misplaced in the document storage unit, improve management efficiency.

\section{Intelligent Document Navigation System Core Technology}

RFID technology is mainly used for document shelf each document storage unit unique identity and document uniqueness logo. After the library is installed, the RFID tag should be placed in the document storage unit of the document rack and registered for location. After the document goes to the museum, the staff first put the RFID tag into the literature, and then further processing and classification of the literature, and then use the system connected with the RFID tag reader to the 
literature and the location of the document associated with the library.

ArcGIS technology is mainly used for the storage and management of library spatial data. The system will be the library floor of the functional area of the composition of the coordinates of the library, the library of the library frame storage unit coordinates, and coordinate points associated with the number of spatial data stored in the ArcGIS database-related data table, based on SharpMap technology to carry out intelligent literature The navigation service establishes the data foundation.

After obtaining the user input document code, the first step is to display the floor number information according to the location of the document where the document is located. The second step is to extract the coordinate information of the composition points of each function area according to the floor number, and generate the floor plan of the floor and highlight the document The third is to extract the coordinates of the composition points of the functional area according to the functional area of the location of the document where the document is located and the coordinates of the points of each document frame to form the position of the collection and the position of the position of the frame Show information on the location of the document.

The above three steps are completed automatically by the system, the system generated prompt information and plane position diagram to the reader clearly shows the literature of the collection information. In the context of document rack management, the administrator uses the RFID tag reader connected to the system to read the document frame storage unit RFID tag, and then read the library of the document storage unit RFID tags, the system will read the data corresponding to the relationship And the system to establish the correct relationship between the corresponding data, you can put the wrong location of the literature information to the administrator.

\section{Conclusion}

GIS and SharpMap are the core technologies of intelligent document navigation system. The intelligent navigation mode fully exploits the potential of RFID technology, ArcGIS technology and SharpMap technology, and constructs the library intelligent document navigation system. The application of the system in the library can further improve the library document management and information service efficiency, strengthen the library information service function, and strengthen the library information security center status.

\section{Acknowledgement}

This paper is one of the research results of "Research on Document Information Resources Sharing System of Guangzhou University Libraries Based on GIS" (Project No. GD14XTS04), which is the "Twelfth Five - Year Plan" of Guangdong Province 's Philosophy and Social Science in 2014.

\section{References}

[1] Wang Jianzhong. Library service in the GIS research status and prospects [J]. Library Work and Research. 2015 (04): 26-28.

[2] WU Qinshu. Multi-level Service System of Cloud GIS [J]. Surveying and Mapping Science and Technology. 2015 (08): 28-32.

[3] WU Weihong. Study on Spatial Distribution of Book Circulation Point Based on GIS-SCENARIO - Taking Suicheng County Central City as an Example [J]. Library Tribune. 2013 
(03): 19-24.

[4] Long Fengming, Li Chengming, YuanXuewang. Application of Task-oriented GIS Service [J]. Bulletin of Surveying and Mapping. 2012 (10): 92-95

[5] Zhou Wenfang. Library digital management-GIS and RFID integration [J]. Information Science. 2010 (07): 1016-1019. 